Canad. Math. Bull. Vol. 66 (1), 2023, pp. 156-165

http://dx.doi.org/10.4153/S0008439522000170

(C) The Author(s), 2022. Published by Cambridge University Press on behalf of The

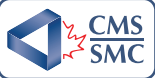

Canadian Mathematical Society. This is an Open Access article, distributed under the terms of the Creative Commons Attribution licence (https://creativecommons.org/licenses/by/4.0/), which permits unrestricted re-use, distribution, and reproduction in any medium, provided the original work is properly cited.

\title{
On a topological Ramsey theorem
}

\author{
Wiesław Kubiś@ and Paul Szeptycki
}

Abstract. We introduce natural strengthenings of sequential compactness, the $r$-Ramsey property for each natural number $r \geq 1$. We prove that metrizable compact spaces are $r$-Ramsey for all $r$ and give examples of compact spaces that are $r$-Ramsey but not $(r+1)$-Ramsey for each $r \geq 1$ (assuming Continuum Hypothesis $(\mathrm{CH})$ for all $r>1$ ). Productivity of the $r$-Ramsey property is considered.

\section{Introduction}

Let $K$ be a compact space, and let $r$ be a positive integer. Following [2], we say that a function $f:[S]^{r} \rightarrow K$ converges to $p \in K$ if for every neighborhood $U$ of $p$, there is a finite set $F$ such that $f\left([S \backslash F]^{r}\right) \subseteq U$. Once this happens for some $p$, we say that $f$ is convergent.

This notion, for $r=2$, was introduced in [2] where the special case of our Theorem 2.1 was stated and proved. Their main motivation was to obtain idempotents in compact semigroups $K$ as limits of certain functions $f:[\omega]^{2} \rightarrow K$. We show that a more general notion of a space satisfying the $r$-Ramsey property (Definition 2.1) given below is a quite natural strengthening of sequential compactness, and the main motivation of this paper is to prove some basic facts about this class of spaces and describe some examples showing that $r$-Ramsey can be strictly weaker than $(r+1)$ Ramsey.

Our topological terminology is standard, and basic definitions and notions can be found in [3]. Set-theoretic notation and terminology, including some background on Ramsey's theorem, can be found in [5], and for a more detailed analysis of almost disjoint families, $\Psi$ spaces, and the ideals $\mathrm{FIN}^{n}$, we refer the reader to [4].

\section{A sequential Ramsey theorem}

Theorem 2.1 Let $(K, \rho)$ be a compact metric space, and let $f:[\omega]^{r} \rightarrow K$ be an arbitrary function, where $r>0$ is a natural number. Then there exists an infinite set $B \subseteq \omega$ such that $f \uparrow[B]^{r}$ converges to some element of $K$.

Received by the editors December 15, 2021; revised February 20, 2022, accepted February 26, 2022.

Published online on Cambridge Core March 7, 2022.

Research of the first author is supported by EXPRO 20-31529X grant (Czech Science Foundation). Research of the second author is supported by the NSERC Discovery Grant program.

AMS subject classification: 54A20, 54D30, 03E02, 03E17.

Keywords: Sequentially compact, Ramsey's theorem, almost disjoint families. 
Proof For each $n \in \omega$, choose a finite cover $\mathcal{U}_{n}$ of $K$ consisting of balls of radius $2^{-n}$. Then $\mathcal{U}_{n}$ induces a finite coloring of $[\omega]^{r}$. Inductively, choose infinite sets $\omega \supseteq$ $A_{0} \supseteq A_{1} \supseteq \cdots$ such that $A_{n}$ is monochromatic for the coloring induced by $\mathcal{U}_{n}$ (here we have used the classical Ramsey's theorem). Let $B$ be any infinite set such that $B \backslash A_{n}$ is finite for every $n \in \omega$. By compactness, $f \uparrow[A]^{r}$ is convergent.

The result above motivates the following definition.

Definition 2.1 Let $X$ be a topological space, and let $r \in \omega$ be positive. We shall say that $X$ has the $r$-Ramsey property (or $X$ is an $r$-Ramsey space) if for every function $f:[\omega]^{r} \rightarrow X$, there exist $p \in X$ and an infinite set $B \subseteq \omega$ such that $f \uparrow[B]^{r}$ converges to $p$. We shall say that $X$ has the Ramsey property if it has the $r$-Ramsey property for every positive $r \in \omega$. We will say that the restriction $f \uparrow B$ is a convergent subsequence off.

Recall that a topological space $X$ is sequentially compact if every sequence in $X$ has a convergent subsequence, and so the 1-Ramsey property is just sequential compactness.

Proposition 2.2 Every space with the r-Ramsey property has the $(r-1)$-Ramsey property, whenever $r>1$. In particular, every space with the $r$-Ramsey property for some $r>0$ is sequentially compact.

Proof Assume $X$ has the $r$-Ramsey property, and fix $g:[\omega]^{r-1} \rightarrow X$. Define $f[\omega]^{r} \rightarrow X$ by setting $f(s)=g(s \backslash \max s)$. Let $A \in[\omega]^{\omega}$ be such that $f \uparrow[A]^{r}$ converges to $p \in X$.

Fix a neighborhood $U$ of $p$. There is $F \in[A]^{<\aleph_{0}}$ such that $f(t) \in U$ whenever $t \subseteq$ $A \backslash F$ and $|t|=r$. Thus, if $s \in[A \backslash F]^{r-1}$, and $k$ is any element of $A$ above $s$, then $g(s)=$ $f(s \cup\{k\}) \in U$.

It is useful to note that if $f:[S]^{r} \rightarrow X$ converges in a sequentially compact $X$, then $f$ has a somewhat nice canonical subsequence.

Definition 2.2 A function $f:[S]^{r} \rightarrow X$ being $r$-nice is defined recursively.

A $f:[S]^{1} \rightarrow X$ is 1-nice if it is convergent. For $r>1, f:[S]^{r} \rightarrow X$ is $r$-nice if:

(1) $f$ is convergent to, say, $x$,

(2) for every $s \in[S]^{r-1}$, the sequence $\{f(s \cup\{n\}): n \in S \backslash s\}$ is convergent to a point $x_{s}$, and

(3) $g:[S]^{r-1}$ defined by $g(s)=x_{s}$ is $(r-1)$-nice.

Note that the fact that $g$, as defined in the definition, is convergent and converges to $x$ follows from the convergence of $f$ to $x$.

Lemma 2.3 If $X$ is sequentially compact, then any convergent $f:[S]^{r} \rightarrow X$ has an $r$-nice convergent subsequence. 
Proof By induction on $r$. If $r=1$, then this follows because $X$ is sequentially compact. Fix $r>1$ and $f:[S]^{r} \rightarrow X$ convergent to some point $x \in X$. Enumerate $[S]^{r-1}$ as $\left\{s_{k}: k \in \omega\right\}$. Define $S_{k}$ recursively. $S_{0} \subseteq S$ is such that $\left\{f(s \cup\{n\}): n \in S_{0} \backslash s_{0}\right\}$ converges to $x_{s_{0}}$. Moreover, in general, $S_{k+1} \subseteq S_{k}$ is chosen such that $\left\{f\left(s_{k} \cup\{n\}\right)\right.$ : $\left.n \in S_{k+1} \backslash s_{k}\right\}$ converges to some $x_{s_{k+1}}$.

Now, take $T$ to be a pseudo-intersection of the $\left\{S_{n}: n \in \omega\right\}$ (i.e., a subset of $\omega$ such that $T \backslash S_{n}$ is finite for each $n$ ) and note that $g:[T]^{r-1} \rightarrow X$ defined by $g(s)=$ $x_{s}$ is defined, and, because $f$ converges to $x$, so $g$ also converges to $x$. Therefore, by our induction hypothesis, $g$ has an $(r-1)$-nice subsequence, and the lemma is proved.

It is easy to prove by induction that the closure of the image of an $r$-nice convergent $f$ is countable and so we have the following corollary:

Corollary 2.4 Assume $X$ is a space with the $r$-Ramsey property, where $r \geq 1$. If $f$ : $[\omega]^{r} \rightarrow X$, then $f$ has a convergent subsequence $f \uparrow[T]^{r}:[T]^{r} \rightarrow X$ such that the closure of $f\left([T]^{r}\right)$ is countable.

\section{Spaces with the Ramsey property}

It is clear that the class of all topological spaces with the Ramsey property is stable under closed subspaces and continuous images. The same applies to the $r$-Ramsey property. In order to see that there are arbitrarily large spaces with the Ramsey property, let $\kappa$ be any cardinal and consider the $\Sigma$-product

$$
\Sigma(\kappa)=\left\{x \in[0,1]^{\kappa}:|\{\alpha: x(\alpha) \neq 0\}| \leq \aleph_{0}\right\} .
$$

Note that for any cardinal $\kappa$, the closure of every countable subset of $\Sigma(\kappa)$ is compact and metrizable; therefore, $\Sigma(\kappa)$ has the Ramsey property. More generally, all monolithic countably compact spaces have the Ramsey property (recall that a space $X$ is monolithic if the closure of every countable subset of $X$ is second countable).

Recall that the unboundedness number $\mathfrak{b}$ is the minimal cardinality of a family $\mathcal{F} \subseteq$ $\omega^{\omega}$ which is unbounded with respect to the almost domination $<^{*}$, where $f<^{*} g$ if $f(n)<g(n)$ for all but finitely many $n \in \omega$.

Theorem 3.1 Every sequentially compact space of character $<\mathfrak{b}$ has the Ramsey property.

Proof Suppose $X$ is a sequentially compact space of character $<\mathfrak{b}$. We use induction on $r$ to prove that $X$ is $r$-Ramsey for every $r \in \omega$. Sequential compactness says that $X$ is 1-Ramsey. Suppose $r>1$ and we have already proved that $X$ is $(r-1)$-Ramsey. Fix $f:[\omega]^{r} \rightarrow X$.

Given $a \in \omega$, let $f_{a}(s)=f(s \cup\{a\})$, where $s \in[\omega \backslash\{a\}]^{r-1}$.

We construct inductively a sequence $a_{0}<a_{1}<\cdots$ in $\omega$, a sequence $\left\{p_{n}: n>0\right\} \subseteq$ $X$, and a decreasing sequence of infinite sets $\omega=A_{0} \supseteq A_{1} \supseteq \cdots$ such that:

(i) $a_{n} \in A_{n}$ and

(ii) $f_{a_{n}} \uparrow\left[A_{n+1}\right]^{r-1}$ converges to $p_{n}$. 
Suppose $n>0$ and $a_{i}, A_{i}$, and $\left\{p_{i}: i<n-1\right\}$ have been constructed for $i<n$. Using the inductive hypothesis, namely, that $X$ is $(r-1)$-Ramsey, we find $p_{n-1} \in X$ and an infinite set $A_{n} \subseteq A_{n-1}$ such that $f_{a_{n-1}} \uparrow\left[A_{n}\right]^{r-1}$ converges to $p_{n-1}$. We set $a_{n}=$ $\min \left(A_{n} \backslash\left(a_{n-1}+1\right)\right)$.

Now, let $M \in[\omega]^{\omega}$ be such that $\left\{p_{n}: n \in M\right\}$ is convergent to some $p \in X$ (here we have used the sequential compactness of $X$ ). Let

$$
B=\left\{a_{n}: n \in M\right\} .
$$

Re-enumerating $A_{n}$ and $p_{n}$, we may assume that $M=\omega$.

Note that the set $B$ has the following property: Given a neighborhood $U$ of $p$, there is $m(U) \in \omega$ such that $\left\{p_{n}: n \geq m(U)\right\} \subseteq U$. Consequently, for every $n \geq m(U)$, there exists $\phi_{U}(n) \in \omega$ such that $f(s) \in U$ whenever $s \in[B]^{r}$ is such that $\min s=a_{n}$ with $n \geq m(U)$ and $\min \left(s \backslash\left\{a_{n}\right\}\right) \geq \phi_{U}(n)$ (the last fact follows from (ii), because $\left.s \backslash\left\{a_{n}\right\} \subseteq A_{n+1}\right)$. Define $\phi_{U}(n)$ arbitrarily for $n<m(U)$.

Let $\mathcal{U}(p)$ be a fixed local neighborhood base at $p$ such that $|\mathcal{U}(p)|<\mathfrak{b}$. Then $\left\{\phi_{U}: U \in \mathcal{U}(p)\right\} \subseteq \omega^{\omega}$ has cardinality $<\mathfrak{b}$; therefore, we can find a strictly increasing function $\psi \in B^{\omega}$ such that $\phi_{U}<^{*} \psi$ for every $U \in \mathcal{U}(p)$. Now, let $C=\{\psi(n): n \in \omega\}$. We claim that $C$ is as required.

Fix $U \in \mathcal{U}(p)$ and fix $s \in[C]^{r}$ such that $\min s=a_{k}$, where $k>m(U)$ and $\psi(n)>$ $\phi_{U}(n)$ for every $n \geq k$. Then $f(s)=f_{a_{k}}\left(s \backslash\left\{a_{k}\right\}\right) \in U$. This shows that $f \uparrow[B]^{r}$ converges to $p$.

Corollary 3.2 Let X be a sequentially compact space in which the closure of every countable set is first countable. Then $X$ has the Ramsey property.

Corollary 3.3 Every countably compact linearly ordered space has the Ramsey property.

Concerning products, it is known that the product of countably many sequentially compact spaces is sequentially compact. The same proof can be used to show

Theorem 3.4 The product of countably many $r$-Ramsey spaces is $r$-Ramsey.

Proof Suppose we are given $r$-Ramsey spaces $X_{i}$ and

$$
f:[\omega]^{r} \rightarrow \prod_{i \in \omega} X_{i}
$$

Recursively choose sets $B_{0} \supseteq B_{1} \supseteq \cdots$ so that for each $i, \pi_{i} \circ f:\left[B_{i}\right]^{r} \rightarrow X_{i}$ converges to $x_{i}$. Then it is straightforward to see that if $B$ is any pseudo-intersection of the family $\left\{B_{i}: i \in \omega\right\}$, then $f:[B]^{r} \rightarrow \prod_{i \in \omega} X_{i}$ converges to $\langle x(i): i<\omega\rangle$.

The splitting number, $\mathfrak{s}$, can be characterized as the minimal $\kappa$ such that $2^{\kappa}$ is not sequentially compact (see [7]). The analogous cardinal characteristic of the continuum that characterizes the $r$-Ramsey property in Cantor cubes is $\mathfrak{p a r}$ and was introduced by Blass in [1]. 
Definition 3.1 A set $A \subseteq \omega$ is almost homogeneous for a partition $f:[\omega]^{n} \rightarrow 2$ if there is a finite $F \subseteq A$ such that $f$ is constant on $[A \backslash F]^{n}$. Furthermore, $\mathfrak{p a r}_{n}$ denotes the smallest cardinal $\kappa$ such that there is a family of partitions of $[\omega]^{n} \rightarrow 2$ of size $\kappa$ such that no infinite set is almost homogeneous for all of them simultaneously.

First, notice that $\mathfrak{p a r}_{1}$ is just the splitting number $\mathfrak{s}$. Moreover, note that if we consider partitions into some finite number of pieces $k$, instead of two pieces, we obtain the same cardinal. Moreover, the following theorem of Blass shows that for all $n \geq 2$, we have that $\mathfrak{p a r}_{n}=\mathfrak{p a r}_{2}$.

Theorem $3.5[1] \quad$ For each $n \geq 2, \mathfrak{p a r}_{n}=\min \{\mathfrak{b}, \mathfrak{s}\}$.

Now, we prove that the minimal $\kappa$ such that $2^{\kappa}$ is not $r$-Ramsey is precisely $\mathfrak{p a r}_{2}$.

Theorem 3.6 For each $\kappa<\mathfrak{p a r}_{2}, 2^{\kappa}$ is $r$-Ramsey for all $r \in \omega$. In addition, $2^{\mathfrak{p a r}_{2}}$ is not 2-Ramsey.

Proof Fix $\kappa<\mathfrak{p a r}_{2}$ and fix $f:[\omega]^{r} \rightarrow 2^{\kappa}$. For each $\alpha<\kappa$, let $f_{\alpha}=\pi_{\alpha} \cdot f$. By the definition of $\kappa<\mathfrak{p a r}_{2}=\mathfrak{p a r}_{r}$, there is $B \subseteq \omega$ and for each $\alpha$ an $i_{\alpha}$ such that for every $\alpha<\kappa$, there is a finite set $F$ such that $f_{\alpha}$ is constant with value $i_{\alpha}$ on $[B \backslash F]^{r}$. This just means that $f:[B]^{r} \rightarrow 2^{\kappa}$ converges to $\left(i_{\alpha}\right)_{\alpha \in \kappa}$ as required.

To complete the proof, to see that $2^{\kappa}$ is not 2-Ramsey for $\kappa=\mathfrak{p a r}_{2}$, fix a family $\left\{f_{\alpha}: \alpha<\kappa\right\}$ so that no $B \subseteq \omega$ is almost homogeneous for all functions $f_{\alpha}$. Taking the product function $f=\prod f_{\alpha}$ we have, as above, that for no $B$ can $f:[B]^{2} \rightarrow 2^{\kappa}$ converge.

Corollary 3.7 If $\mathfrak{b}<\mathfrak{s}$, then $2^{\mathfrak{b}}$ is sequentially compact but not 2-Ramsey.

\section{Examples}

In this section, we give some examples of spaces with the $k$-Ramsey property that do not have the $(k+1)$-Ramsey property. The first example of a 1-Ramsey (i.e., sequentially compact) not 2-Ramsey is in ZFC, but for larger $k$, we assume the Continuum Hypothesis $(\mathrm{CH})$. All of the examples are of the form $K(\mathcal{A})=\alpha(\Psi(\mathcal{A}))$, the Alexandrov-Urysohn compactum formed by taking the one-point compactification of the $\Psi$-space determined by an almost disjoint family $\mathcal{A}$ of infinite subsets of $\omega$. Infinite sets $a, b \subseteq \omega$ are said to be almost disjoint if $a \cap b$ is finite. If $\mathcal{A}$ is a family of subsets of $\omega$ that are pairwise almost disjoint, then the space $\Psi(\mathcal{A})=\omega \cup \mathcal{A}$ where the points of $\omega$ are isolated and neighborhood base for $a \in \mathcal{A}$ is given by sets of the form $\{a\} \cup(a \backslash n)$. It is well known that $K(\mathcal{A})$, the one-point compactification of $\Psi(\mathcal{A})$, is sequentially compact (see [4] for more on almost disjoint families and $\Psi$-spaces).

If $\mathcal{A}$ is a maximal almost disjoint (mad) family, then $K(\mathcal{A})$ is not even 2-Ramsey.

Example 4.1 Let $\mathcal{A}$ be an infinite maximal almost disjoint family on $[\omega]^{\omega}$. Then the Alexandrov-Urysohn compactum $K(\mathcal{A})$ is a compact and sequentially compact space failing the 2-Ramsey property. 
Proof For convenience, we assume that $\mathcal{A} \subseteq[\omega \times \omega]^{\omega}$ and $\{n\} \times \omega \in \mathcal{A}$ for each $n \in \omega$. Thus,

$$
K(\mathcal{A})=(\omega \times \omega) \cup\left\{p_{A}: A \in \mathcal{A}\right\} \cup\{\infty\},
$$

where we write $p_{n}$ instead of $p_{\{n\} \times \omega}$. Then $\omega \times \omega$ is the set of isolated points, $K(\mathcal{A}) \backslash\{\infty\}$ is locally compact, and a basic neighborhood of $p_{A}$ is $\left\{p_{A}\right\} \cup(A \backslash F)$, where $F \in[A]^{<\omega}$.

Let $f:[\omega]^{2} \rightarrow K(\mathcal{A})$ be defined by $f(\{k, l\})=\langle k, l\rangle$, where $k<l$. Now, it is easy to see that this $f$ witnesses the failure of 2-Ramsey, because for any $S \subseteq \omega$ infinite, the closure of $f\left([B]^{2}\right)$ in $\Psi(\mathcal{A})$ is infinite, and because $\mathcal{A}$ is mad, it follows that the closure is uncountable. Therefore, by Corollary $2.4, K(\mathcal{A})$ cannot be 2 -Ramsey.

We now describe, for each $r>1$, an $r$-Ramsey not $(r+1)$-Ramsey compact space of the form $K(\mathcal{A})$. We state a few lemmas about these properties in these types of spaces.

Lemma 4.2 For any almost disjoint family $\mathcal{A}$ on a countable set I of isolated points, $K(\mathcal{A})$ is $r$-Ramsey if and only if for any $f:[\omega]^{r} \rightarrow I$, there is $B \subseteq \omega$ such that $f \uparrow[B]^{r}$ converges.

Proof The property is clearly necessary. For sufficiency, note that for any $f:[\omega]^{r} \rightarrow$ $K(\mathcal{A})$, one can find $B$ such that either $f\left([B]^{r}\right) \subseteq \mathcal{A}$ (in which case, because any infinite subset converges, one can easily find $B^{\prime} \subseteq B$ witnessing $r$-Ramsey) or $f\left([B]^{r}\right) \subseteq I$, as required.

Lemma 4.3 $K(\mathcal{A})$ is $r$-Ramsey iffor every $f:[\omega]^{r} \rightarrow I$, there is an infinite $B \subseteq \omega$ such that

$$
\mathcal{A} \uparrow f\left([B]^{r}\right)=\left\{p \in \mathcal{A}: p \cap f\left([B]^{r}\right) \text { is infinite }\right\}
$$

is of size less than $\mathfrak{b}$.

Proof If we can find such a $B$, then the closure of the subset $f\left([B]^{r}\right)$ in $K(\mathcal{A})$ has countable character at all points of $\mathcal{A}$ and character less than $\mathfrak{b}$ at $\infty$, and so by Theorem 3.1, this subspace is $r$-Ramsey, and so we can find $B^{\prime} \subseteq B$ on which $f$ converges.

We now turn to a construction of an $r$-Ramsey example of an AlexandrovUrysohn compactum that is not $(r+1)$-Ramsey.

We first need some basic properties of the Fubini product of the ideal FIN. Recall that FIN is the ideal of finite subsets of $\omega$ and, for each $n>1, \operatorname{FIN}^{n}$ is the ideal on $\omega^{n}$ defined recursively by

$$
A \in \operatorname{FIN}^{n} \text { if }\left\{\bar{k} \in \omega^{n-1}:\{j: \bar{k}-j \in A\} \text { is infinite }\right\} \in \operatorname{FIN}^{n-1} .
$$

The following lemma is easily proved using the definition. 
Lemma 4.4 For any $X \subseteq \omega^{n}$, if $X \notin F I N^{n}$, then there is a $T \subseteq X$ such that $T$ forms an everywhere $\omega$-splitting tree. That is, letting $T_{i}=\{x \uparrow i: x \in T\}$, for each $i<n$ and each $s \in T_{i},\left\{t \in T_{i+1}: t \uparrow i=s\right\}$ is infinite.

Now, we need the following lemma.

Lemma 4.5 For any function $f:[\omega]^{n} \rightarrow \omega^{n+1}$, there is a $B \subseteq \omega$ infinite such that $f\left([B]^{n}\right) \in F I N^{n+1}$.

Proof By induction on $n$. When $n=1$, we can find $B$ such that $f(B)$ is either contained in a column $\{k\} \times \omega$ or is a partial function. In either case, $f(B) \in \mathrm{FIN}^{2}$.

For the inductive step, fix $f:[\omega]^{n} \rightarrow \omega^{n+1}$ and fix $k_{0} \in \omega$ arbitrary. Define $f_{k_{0}}$ : $\left[\omega \backslash k_{0}+1\right]^{n-1} \rightarrow \omega^{n+1}$ by $f_{k_{0}}(s)=f\left(\left\{k_{0}\right\} \cup s\right)$. By our inductive assumption, there is an infinite $B_{1} \subseteq \omega \backslash k_{0}+1$ such that:

$(*)$ the projection of $f_{k_{0}}\left(\left[B_{1}\right]^{n-1}\right)$ onto any $n$ coordinates of $\omega^{n+1}$ is in $\mathrm{FIN}^{n}$.

Let $k_{1}=\min \left(B_{1}\right)$ and continue recursively constructing $\left\{k_{i}, B_{i}\right\}$ so that for each $i$, $k_{i}=\min \left(B_{i}\right)$ and

(*) the projection of $f_{k_{i}}\left(\left[B_{i+1}\right]^{n-1}\right)$ onto any $n$ coordinates of $\omega^{n+1}$ is in FIN ${ }^{n}$.

Now, let $B=\left\{k_{i}: i \in \omega\right\}$. Since $(\omega+1)^{n+1}$ is $r$-Ramsey for all $r$, we may, by possibly shrinking $B$, assume that $f:[B]^{n} \rightarrow(\omega+1)^{n+1}$ converges to some $x$.

CASE 1. $x(i)=\omega$ for all $i<n+1$. In this case, if $f\left([B]^{n}\right)$ is not in $\operatorname{FIN}^{n+1}$, then there is an everywhere $\omega$-splitting tree $T \subseteq f\left([B]^{n}\right)$ witnessing this. Fix $z \in T$ and consider $k=z(0)$. Since $f:[B]^{n} \rightarrow(\omega+1)^{n+1}$ converges to $x$, there is an $N$ such that

$$
f\left([B \backslash N]^{n}\right) \subseteq(\omega \backslash k+1)^{n+1} .
$$

Therefore, $f\left([B \backslash N]^{n}\right)$ is disjoint from $T^{\prime}=\{s \in T: s(0)<N\}$. Note that the projection of $T^{\prime}$ onto $\omega^{n+1 \backslash\{0\}}$ is not in $\mathrm{FIN}^{n}$. Furthermore, $T^{\prime}$ is covered by the set $f\left(\left\{s \in[B]^{n}: \min s<N\right\}\right)$, but for each $k_{i}<N$, the projection of the sets $f\left(\left\{s \in[B]^{n}\right.\right.$ : $\left.\min s=k_{i}\right\}$ ) onto $\omega^{n+1 \backslash\{0\}}$ is in $\mathrm{FIN}^{n}$, a contradiction.

CASE 2 . There is $i$ such that $x(i) \neq \omega$. In this case, there is some $N$ such that

$$
f\left([B \backslash N]^{n}\right) \subseteq\left\{z \in(\omega)^{n+1}: z(i)=x(i)\right\} .
$$

In addition, this completes the proof because $\left\{z \in(\omega)^{n+1}: z(i)=x(i)\right\}$ is in FIN $^{n+1}$.

Now, fix $r>1$ and we will need to assume $\mathrm{CH}$. We build an almost disjoint family $\mathcal{A}$ on $\omega^{r+1}$ so that for the function $G:[\omega]^{r+1} \rightarrow \omega^{r+1}$ defined by $G(x)=\langle x(0), \ldots, x(r)\rangle$ where $x=\{x(0), \ldots, x(r)\}$ is the increasing enumeration of $x, G$ will have no convergent subsequence $B$.

To simplify some notation for any $B$, let

$$
B^{\uparrow n}=\left\{\bar{k} \in B^{n}: \bar{k}(i)<\bar{k}(i+1) \text { for all } i<n-1\right\} .
$$

Lemma 4.6 Suppose $\mathcal{A}$ is an almost disjoint family on $\omega^{r+1}$. For the function $G$ defined as above, $B$ is a convergent subsequence for $G$ with limit $\infty$ in $K(\mathcal{A})$ if and only if for every $a \in \mathcal{A}$, there is an $n$ such that $a \cap(B \backslash n)^{\uparrow r+1}=\varnothing$. 
Proof Directly from the definitions.

Theorem 4.7 Assume CH. For each $r>1$, there is an almost disjoint family $\mathcal{A}$ on $\omega$ such that $K(\mathcal{A})$ is $r$-Ramsey but not $(r+1)$-Ramsey.

Proof We will construct $\mathcal{A}=\left\{a_{\alpha}: \alpha<\omega_{1}\right\}$ an almost disjoint family on $\omega^{r+1}$ by defining each $a_{\alpha}$ by recursion on $\alpha$. We start by letting $\left\{a_{n}: n \in \omega\right\}$ be an enumeration of the disjoint family

$$
\left\{\{\bar{k}\} \times \omega: \bar{k} \in \omega^{r}\right\} .
$$

Note that each $a_{n} \in \mathrm{FIN}^{n+1}$ and

(*) any $x$ that is almost disjoint from all the $a_{n}$ is also in $\mathrm{FIN}^{n+1}$.

We enumerate as $\left\{B_{\alpha}: \omega \leq \alpha<\omega_{1}\right\}$ all infinite subsets of $\omega$ and fix an enumeration

$$
\left\{f_{\alpha}: \omega \leq \alpha<\omega_{1}\right\}=\left(\omega^{r+1}\right)^{[\omega]^{r}} \text {. }
$$

Recall our plan that $G:[\omega]^{r+1} \rightarrow \omega^{r+1}$ should have no convergent subsequence.

Suppose that at some stage $\alpha$ of the construction, we have defined $\left\{a_{\beta}: \beta<\alpha\right\}$ and infinite sets $\left\{X_{\beta}: \omega \leq \beta<\alpha\right\}$ such that:

(1) For all $\omega \leq \beta<\alpha, f_{\beta}\left(\left[X_{\beta}\right]^{r}\right) \in \operatorname{FIN}^{n+1}$.

(2) For all $\beta<\gamma<\alpha, a_{\beta} \cap a_{\gamma}$ is finite.

(3) For all $\omega \leq \beta<\gamma<\alpha,\left(f_{\beta}\left(\left[X_{\beta}\right]^{r}\right)\right) \cap a_{\gamma}$ is finite.

(4) For all $\omega \leq \beta<\alpha$ and all $n<\omega, a_{\beta} \subseteq^{*} G\left(\left[B_{\beta} \backslash n\right]^{r+1}\right)$.

To define $a_{\alpha}$ and $X_{\alpha}$, note first, by clause (*) and (1) above, it follows that

$$
\mathcal{H}=\left\{f_{\beta}\left(\left[X_{\beta}\right]^{r}\right): \beta<\alpha\right\} \cup\left\{a_{\beta}: \beta<\alpha\right\} \subseteq \operatorname{FIN}^{n+1} .
$$

Therefore, the family

$$
\left\{G\left(\left[B_{\alpha} \backslash n\right]^{r+1}\right) \backslash H: n \in \omega, H \in \mathcal{H}\right\}
$$

has an infinite pseudo-intersection. Let $a_{\alpha}$ be any such pseudo-intersection, and it directly follows that $a_{\alpha}$ satisfies the inductive hypotheses (2)-(4). Therefore, we need only define $X_{\alpha}$ to satisfy (1), but that there is such an $X_{\alpha}$ follows from Lemma 4.5.

This completes the construction of $\mathcal{A}=\left\{a_{\alpha}: \alpha<\omega_{1}\right\}$. To see that it is $r$-Ramsey, by Lemma 4.2, we need only consider functions $f:[\omega]^{r} \rightarrow \omega^{r+1}$, and each $\operatorname{such} f$ appears as an $f_{\alpha}$. For each $\alpha$ by inductive hypothesis (3), we have that

$$
\left\{a \in \mathcal{A}: a \cap f_{\alpha}\left(\left[X_{\alpha}\right]^{r}\right) \text { is infinite }\right\}
$$

is countable. And so by Lemma 4.3, it follows that $K(\mathcal{A})$ is $r$-Ramsey. On the other hand, to see that $G$ has no convergent subsequence, first note that if any $B_{\alpha}$ were to form a convergent subsequence for $G$, then it would need to converge to $\infty$. Indeed, for all $n$, we have $\left\{a_{k}:\left|a_{k} \cap G\left(\left[B_{\beta} \backslash n\right]^{r+1}\right)\right|=\aleph_{0}\right\}$ is infinite and so for no $n$ is $G\left(\left[B_{\beta} \backslash n\right]^{r+1}\right) \subseteq^{*} a_{\alpha}$ for any $\alpha<\omega_{1}$. And now, inductive hypothesis (4) implies, by Lemma 4.6, that no $B_{\alpha}$ is a convergent subsequence for $G$ and so $K(\mathcal{A})$ is not $(r+1)$ Ramsey. 


\section{Questions}

We finish with the following questions.

Question 1 Does there exist a space with the $r$-Ramsey property and without the $(r+1)$-property assuming only ZFC? Here $r>1$.

Note that all our examples of $r$-Ramsey and not $(r+1)$-Ramsey spaces (which required $\mathrm{CH}$ ) are Fréchet-Urysohn, but the ZFC example that was not 2-Ramsey, being a $K(\mathcal{A})$ where $\mathcal{A}$ is mad, is not Fréchet-Urysohn. However, a similar example could be constructed from a completely separable mad family. The main idea is to start with a completely separable mad family on $\omega \times \omega$ as in the construction of Example 4.1. Then, deleting an infinite set from each $A \in \mathcal{A}$ other than the fixed columns $\{n\} \times \omega$ will still give an example that fails to be 2 -Ramsey, but it will be FréchetUrysohn because it is nowhere mad (see [4] for the definitions of completely separable and nowhere mad). So we have the following example.

Example 5.1 Assuming the existence of a completely separable mad family, there is an almost disjoint $\mathcal{A}$ such that $K(\mathcal{A})$ is Fréchet-Urysohn and not 2-Ramsey.

Although the existence of a completely separable mad family is a relatively weak one (e.g., it follows from $\mathfrak{c}<\aleph_{\omega}$ or $\mathfrak{s} \leq \mathfrak{a}$; see [4]), we ask the following question.

Question 2 Does there exist a ZFC example of a Fréchet-Urysohn compact space without the Ramsey property?

We know that a product of any countable family of $r$-Ramsey spaces is $r$-Ramsey, and we have characterized when $2^{\kappa}$ is $r$-Ramsey. Moreover, $\mathfrak{h}$ is the minimal $\kappa$ such that a product of fewer than $\kappa$ many sequentially compact spaces is sequentially compact [6], and we conjecture the same holds for $r$-Ramsey. The cardinal $\mathfrak{h}$ has many equivalent formulations, but the one most relevant here is that $\mathfrak{h}$ is the minimal cardinality of a collection $\mathcal{F}$ of mad families on $\omega$, so that any infinite $X \subseteq \omega$ has infinite intersection with at least two elements of some family from $\mathcal{F}$ (see [1]). This characterization also makes clear that $\mathfrak{h} \leq \mathfrak{p a r} \mathfrak{r}_{2}$.

The proof that the product of fewer than $\mathfrak{h}$ sequentially compact spaces is sequentially compact also shows the same for $r$-Ramsey, but the family of sequentially compact spaces whose product is not sequentially compact given in [6] are, in fact, Alexandrov-Urysohn compacta over mad families, and, as we have seen, these spaces are not even 2-Ramsey. Therefore, if $\mu$ is the minimal cardinal for productivity of the class of 2-Ramsey spaces, then $\mathfrak{h} \leq \mu$ and we conjecture that $\mu=\mathfrak{h}$.

Question 3 Characterize the minimal cardinal $\kappa$ satisfying the product of fewer than $\kappa$ many $r$-Ramsey spaces is always $r$-Ramsey.

Acknowledgment The first author would like to thank Henryk Michalewski for bringing this topic and in particular for pointing out reference [2]. The second author 
would like to thank the first author and the Institute of Mathematics of the Czech Academy of Sciences for support during his visit when this research was conducted.

\section{References}

[1] A. Blass, Combinatorial cardinal characteristics of the continuum, Handbook of set theory. In: M. Foreman and A. Kanamori (eds.), Springer, 2010, pp. 395-489.

[2] M. Bojańczyk, E. Kopczyński, and S. Toruńczyk, Ramsey's theorem for colors from a metric space. Semigroup Forum 85(2012), 182-184.

[3] R. Engelking, General topology, Sigma Series in Pure Mathematics, 6, Heldermann, Berlin, 1989.

[4] M. Hrušak, Almost disjoint families and topology, Recent progress in general topology III. Ed. K.P. Hart, J. van Mill, and P. Simon (eds.), Springer, 2014, pp. 601-638.

[5] K. Kunen, Set theory, Studies in Logic, 34, College Publications, London, 2013.

[6] P. Simon, Products of sequentially compact spaces. Rend. Istit. Mat. Univ. Trieste 25(1993), 447-450.

[7] E. K. van Douwen, The integers and topology, The handbook of set-theoretic topology. K. Kunen and J. Vaughan (eds.), Elsevier, 1984, pp. 111-168.

Institute of Mathematics, Czech Academy of Sciences, 11720 Staré Město, Czechia

e-mail: kubis@math.cas.cz

Department of Mathematics and Statistics, York University, Toronto, Ontario M3J 1P3, Canada

e-mail: szeptyck@yorku.ca 